\title{
Performance-Based Comparative Assessment of Open Source Web Vulnerability Scanners
}

\author{
Mansour Alsaleh, ${ }^{1}$ Noura Alomar, ${ }^{2}$ Monirah Alshreef, \\ Abdulrahman Alarifi, ${ }^{1}$ and AbdulMalik Al-Salman ${ }^{2}$ \\ ${ }^{1}$ King Abdulaziz City for Science and Technology, Riyadh, Saudi Arabia \\ ${ }^{2}$ College of Computer and Information Sciences, King Saud University, Riyadh, Saudi Arabia \\ Correspondence should be addressed to Mansour Alsaleh; maalsaleh@kacst.edu.sa
}

Received 7 January 2017; Revised 25 March 2017; Accepted 16 April 2017; Published 24 May 2017

Academic Editor: Michele Bugliesi

Copyright (C) 2017 Mansour Alsaleh et al. This is an open access article distributed under the Creative Commons Attribution License, which permits unrestricted use, distribution, and reproduction in any medium, provided the original work is properly cited.

The widespread adoption of web vulnerability scanners and the differences in the functionality provided by these tool-based vulnerability detection approaches increase the demand for testing their detection effectiveness. Despite the advantages of dynamic testing approaches, the literature lacks studies that systematically evaluate the performance of open source web vulnerability scanners. The main objectives of this study are to assess the performance of open source scanners from multiple perspectives and to examine their detection capability. This paper presents the results of a comparative evaluation of the security features as well as the performance of four web vulnerability detection tools. We followed this comparative assessment with a case study in which we evaluate the level of agreement between the results reported by two open source web vulnerability scanners. Given that the results of our comparative evaluation did not show significant performance differences among the scanners while the results of the conducted case study revealed high level of disagreement between the reports generated by different scanners, we conclude that the inconsistencies between the reports generated by different scanners might not necessarily correlate with their performance properties. We also present some recommendations for helping developers of web vulnerabilities scanners to improve their tools' capabilities.

\section{Introduction}

The multilayered architectures of web-based systems and their sophisticated interactions with different types of subsystems increase the number of security flaws that can be exploited by attackers. As emphasized by the Open Web Security Application Project (OWASP) [1], attackers might follow various paths through the digital infrastructure of an organization to find security weaknesses that might cause severe consequences. According to Symantec [2], over 6500 web vulnerabilities were reported in 2014 and twenty percent of them were expected to have critical consequences. Kaspersky also reported that more than 93 million malicious URLs were detected in the first quarter of 2015 [3]. By 2018, web server vulnerabilities are also expected to be ranked as the first in the list of the most serious cybersecurity attacks [4].
Dynamic security testing and static code analysis are ones of the main approaches that are used for detecting web vulnerabilities. Software developers use web vulnerability scanners to automate the process of examining the security of their web-based applications and conduct large-scale security analyses on many different web applications [5]. While automated static code analysis techniques are more effective for detecting security defects (e.g., SQL vulnerabilities) $[6,7]$, the adoption of these techniques is limited compared to the widespread application of automated dynamic security testing tools. One of the reasons that contributed to limiting the adoption of static code analysis by software developers is that it cannot be performed without accessing the source code of the evaluated web-based application, which might not always be accessible [5]. 
There are many commercial tool-based web vulnerability detection approaches for identifying web security defects. IBM Security AppScan [8], HP webInspect [9], Acunetix web vulnerability scanner [10] are all examples of commercial dynamic web vulnerability detection tools. Although these scanners are considered state-of-the-art and were proved to be effective in identifying and analyzing security vulnerabilities, their utilization is limited to the developers who can afford their high prices [11-14]. In addition, a large percentage of the web content is developed by either individual developers or small and medium-sized enterprises that do not follow a secure software development life cycle and thus pay more attention to delivering the functionality of their systems than dealing with system security complexities. Such web applications and systems are vulnerable to various attacks compromising their web servers' code integrity [15]. Due to the high impact of the propagation of security attacks on the web, there is a high demand for facilitating the vulnerability detection activities by providing effective, efficient, and easy to use web vulnerability scanners. As an attempt to fulfill this demand, our study focuses on evaluating the performance of open source web vulnerability scanners and determining whether their cost-effectiveness negatively correlates with their detection capability and accuracy.

In this paper, we conduct a comprehensive evaluation of the performance of a set of open source dynamic web application security testing tools. These tools work by crawling or scanning all web pages within a domain and automating the security testing process by randomly simulating attacking scenarios [16]. Although web security scanners might be effective in identifying security flaws and malicious content, previous studies proved that there are inconsistencies between the results reported by different scanners [17-19]. Further, these tools vary in their performance characteristics, types of detected vulnerabilities, and crawler coverage [1719]. This in turn decreases the level of trustworthiness in web vulnerability scanners and increases the demand for conducting experiments that quantitatively evaluate their quality and accuracy.

Our study started by reviewing some of the currently available open source dynamic security testing tools and choosing four of them based on a predefined selection criteria. After performing a comparative evaluation of the performance of the four chosen scanners, two scanners were chosen to be included in a case study which utilized these tools to detect the security flaws in 140 unique web-based applications. Wapiti, Skipfish, and two versions of Arachni open source scanners were studied in our comparative assessment whereas the experimental evaluation performed in our case study included Wapiti 2.3.0 and Arachni 0.4.3 only.

Of the four evaluated scanners, our results show that Skipfish 2.1 [20] is the fastest. In terms of crawler coverage, however, Arachni 1.0.2 [21] ranks first with a coverage percentage of $94 \%$. We utilized the benchmark provided by the Web Application Vulnerability Scanner Evaluation Project (WAVSEP) [22] to evaluate the accuracy of the four scanners in question. Our findings reveal that the best SQL and XSS vulnerability detection rates were obtained by Arachni 1.0.2 which detected $100 \%$ of the SQL test cases and $87.67 \%$ of the XSS test cases. After scanning a sample of 140 websites using Arachni 0.4.3 and Wapiti 2.3.0 [23], we found that both scanners indicated that at least $21 \%$ of the assessed websites had high-severity security vulnerabilities. Arachni 0.4 .3 and Wapiti 2.3 .0 also found that $12.86 \%$ of the evaluated websites were vulnerable to SQL attacks whereas $11.43 \%$ of them had XSS vulnerabilities. Given that the results of our comparative evaluation did not show significant performance differences (e.g., scanning speed) among the scanners while the results of the conducted case study revealed high level of disagreement between the reports generated by different scanners, we conclude that the inconsistencies between the reports generated by different scanners might not necessarily correlate with their performance properties. Further research is therefore needed to investigate the factors that could degrade the effectiveness of web vulnerability detection scanners and thus lead them to report inconsistent results.

Contributions. We frame the contributions of our work as follows.

(1) Comparative Evaluation of Open Source Web Vulnerability Scanners. After reviewing currently available open source web vulnerability scanners, a comparative evaluation of the security features as well as the performance of four web vulnerability detection tools is conducted. Two versions of Arachni scanner as well as Wapiti and Skipfish scanners are evaluated in terms of speed, crawler coverage, detection accuracy, and extra supported features. The conducted evaluation is based on rigorous performance indicators as well as standard security benchmarks.

(2) Case Study on the Security State of Web-Based Systems. As a second step for evaluating the performance of web vulnerability detection tools, a comprehensive web vulnerability assessment on 140 web-based applications is conducted. Two selected tools were utilized to identify the types, quantities, and degrees of severity of security vulnerabilities that exist in the evaluated web content. The results reported by these scanners are then analyzed, aggregated, compared, and linked to the results obtained from our scanners' comparative evaluation experiment to clearly understand the detection accuracy of these tools in various scenarios.

(3) Design Guidelines. We present some recommendations for helping developers of web vulnerabilities scanners to improve their tools' capabilities.

Organization. We first describe the steps followed to review, compare, and select open source web vulnerability scanners in Section 2. We then present the results of the conducted comparative evaluation in Section 3. In Section 4, we use two web vulnerability scanners to detect the security vulnerabilities in a large collected dataset. Further discussion, design guidelines for tool developers, and the related literature are then presented in Section 5, Section 6, and Section 7, respectively. We present the limitations and future work in Section 8 and conclude in Section 9. 


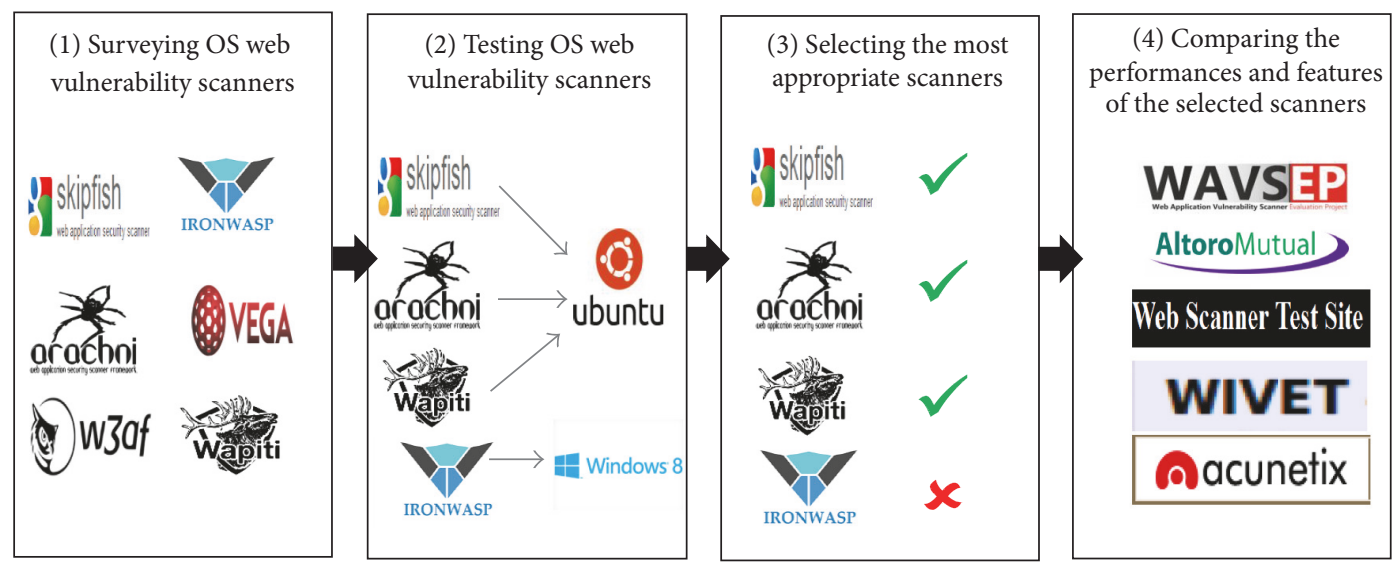

FIGURE 1: Web vulnerability scanners' comparison: research approach.

\section{Web Vulnerability Scanners: Evaluation Approach}

We followed a structured research methodology that focuses on addressing the problem from multiple perspectives. We started by reviewing some popular open source web vulnerability scanning tools based on a predefined criteria. We then randomly chose a set of web-based applications and scanned them using some of the surveyed open source scanners. Three of them were then selected to be included in our detailed open source web vulnerability scanners comparative evaluation. Figure 1 summarizes the web vulnerability scanners and security benchmarks utilized throughout each phase of our evaluation approach. The following subsections further explain the decisions taken throughout the phases of the comparative assessment.

2.1. Web Vulnerability Scanners Selection. The first phase of our study involved conducting an initial survey of the most popular open source web vulnerability assessment tools. Six scanners were evaluated against predefined criteria that consider the performance properties of the scanners as well as their crawler coverage and types of web vulnerabilities they can identify (see Table 1). The open source web vulnerability assessment scanners that were included in our survey are Skipfish, Arachni, Wapiti, IronWASP, Vega [24], and w3af [25].

For each scanner, we investigated the provided security scanning and visualization features, the supported operating systems, and the scanning scope. In order to evaluate these scanners and include the most effective ones in our comparative evaluation, we chose the best four scanners and tested them on some randomly selected websites. These scanners are Wapiti, Arachni, Skipfish, and IronWASP. In the testing phase, Wapiti, Arachni, and Skipfish were installed on Ubuntu 12.04 whereas IronWASP [26] was installed on Windows 8.

Based on the scanning results obtained in the testing phase, two web vulnerability scanners were chosen to be included in the conducted comparative assessment: Wapiti 2.3.0 and Arachni 0.4.3. In fact, after comparing the results reported by the four scanners, we found inconsistencies
TABLE 1: Web vulnerability scanners' comparison: scanners' selection and evaluation criteria.

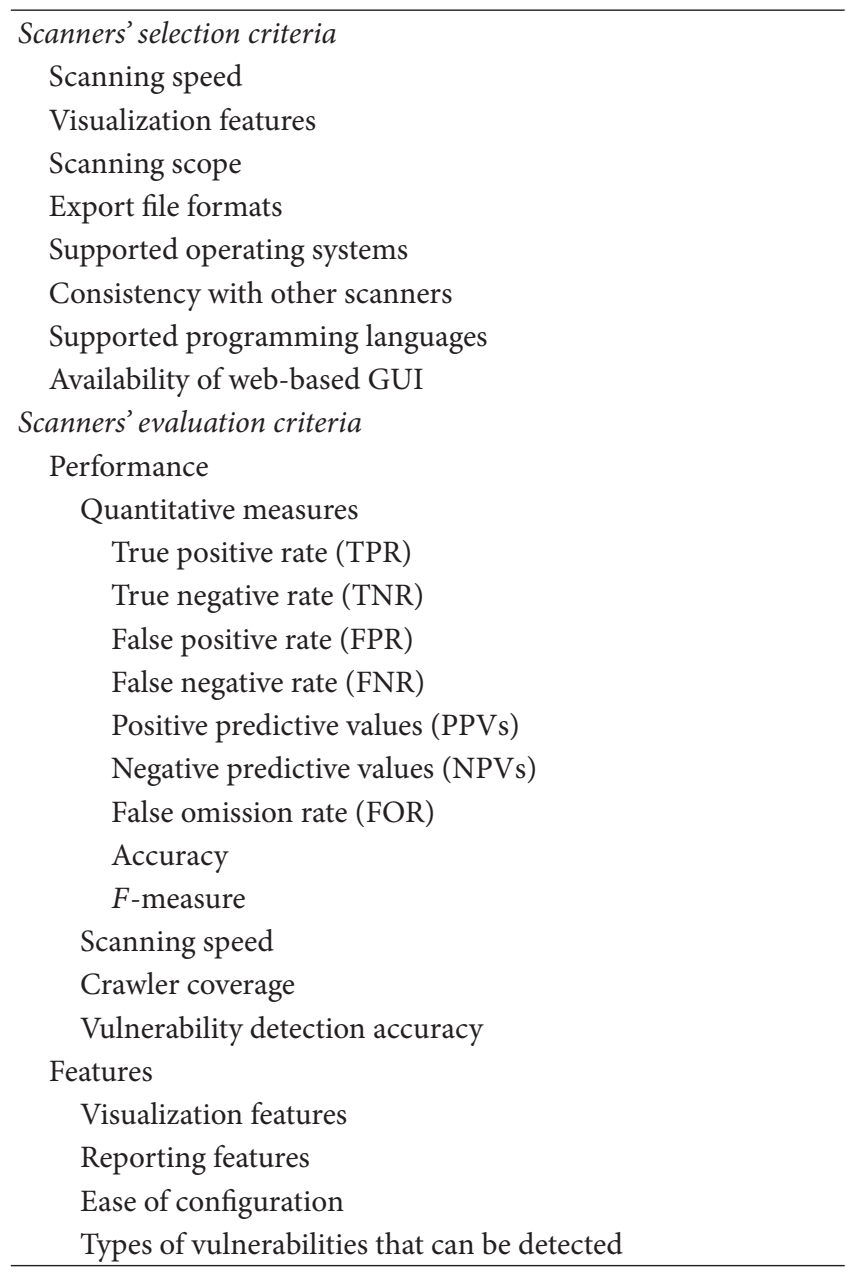

between the types and quantities of security vulnerabilities reported by IronWASP and the other three scanners. Given this and the limited reporting features of IronWASP, we did exclude IronWASP scanner. Skipfish was also not included in the security evaluation conducted in our case study. However, 
TABLE 2: The speed and crawler depth results obtained after scanning the Web Scanner Test Site and the two Acunetix sites.

\begin{tabular}{lccccccc}
\hline Scanner & Version & $\begin{array}{c}\text { webscantest } \\
\text { (HH:MM:SS) }\end{array}$ & $\begin{array}{c}\text { testphp } \\
\text { (HH:MM:SS) }\end{array}$ & $\begin{array}{c}\text { testaspnet } \\
\text { (HH:MM:SS) }\end{array}$ & Rank (speed) & Crawler coverage & Rank (crawler coverage) \\
\hline Arachni & 0.4 .3 & $01: 23: 22$ & $00: 22: 10$ & $01: 05: 33$ & 2 & $19 \%$ & 4 \\
Arachni & 1.0 .2 & $12: 11: 13$ & $00: 41: 34$ & $01: 00: 00$ & 3 & $94 \%$ & 1 \\
Wapiti & 2.3 .0 & $06: 20: 00$ & $01: 30: 00$ & $03: 30: 00$ & 4 & $44 \%$ & 3 \\
Skipfish & 2.1 & $00: 11: 06$ & $00: 27: 40$ & $00: 11: 30$ & 1 & $48 \%$ & 2 \\
\hline
\end{tabular}

we included Skipfish in our comparative evaluation of web vulnerability detection tools in order to compare its speed with those of Wapiti and Arachni scanners.

\subsection{Web Vulnerability Scanners Comparison. As a prestep to} evaluating the security of a sample of 140 distinct web-based applications, we conducted a comparative evaluation of four web vulnerability detection tools which are Arachni 0.4.3, Arachni 1.0.2, Wapiti 2.3.0, and Skipfish 2.10. The aim of this evaluation was to assess the effectiveness of these scanners and decide whether their reported results will accurately reflect the security state of the websites examined in our case study. We evaluated the scanning speed of each tool, their crawler depth, and their vulnerability detection accuracy. Our evaluation criteria also considered the extra features supported by each scanner (e.g., reporting and visualization services) and the types of web vulnerabilities that can be discovered by each scanner. Table 1 summarizes the criteria that were defined to evaluate the chosen web scanners, which covers the essential performance characteristics that allowed us to base our evaluation on rigorous standards and benchmarks.

Three vulnerability test websites were considered for evaluating the speed of each scanner: Web Scanner Test Site [27] and two Acunetix [28] sites. These test websites were fully scanned using the selected four web vulnerability scanners. We then compared the scanning times of the scanners and ranked them accordingly. Similarly, Altoro Mutual [29] and WAVSEP [22] were utilized for assessing the accuracy of the scanners. We also used the Web Input Vector Extractor Teaser (WIVET) [30] benchmarking project to examine the crawling capabilities of the evaluated scanners. To assess the accuracy of each scanner, we calculated (1) the true positive rates (TPRs); (2) the true negative rates (TNRs); (3) the false positive rates (FPRs); (4) the false negative rates (FNRs); (5) the positive predictive values (PPVs); (6) the negative predictive values (NPVs); (7) and the false omission rates (FORs) [31,32]. We also measured the vulnerability detection accuracy of the evaluated scanners and their associated $f$ measures.

\section{Scanners' Comparative Evaluation: Results and Analysis}

After using Arachni 0.4.3, Arachni 1.0.2, Wapiti 2.3.0, and Skipfish 2.10 to scan the chosen test websites, we observed the scanning behaviors of the evaluated scanners and quantitatively compared their speed, detection accuracy, and crawler coverage. We then ranked these scanners accordingly and chose Arachni 0.4.3 and Wapiti 2.3.0 to be included in our case study (as explained in Section 4). In this section, we present and discuss the results of our web vulnerability scanners comparative assessment.

In general, our results show that the speed of the evaluated scanners was relatively acceptable. However, in terms of crawler coverage, the obtained WIVET scores for the four scanners show that $75 \%$ of them covered less than $50 \%$ of the crawled web-based systems. By considering the detection accuracy of the evaluated scanners, we found variations between the scanning results obtained from Altoro Murual and WAVSEP. For example, all the scanners detected at least $77 \%$ of WAVSEP's SQL test cases whereas $75 \%$ of them detected at most 38\% of Altoro Mutual's SQL test cases. However, this might be due to differences between the security properties examined by WAVSEP's and Altoro Mutual's test cases. We also observed variations in number and types of web vulnerabilities that each scanner can detect. However, all the chosen web scanners were able to discover basic security defects including database injection attacks, XSS vulnerabilities, and misconfiguration attacks. Further, no difficulties were faced in understanding the functionality of the evaluated scanners, as all of them provide sufficient documentation in their corresponding websites. The following subsections detail the results of the conducted comparative assessment.

3.1. Speed. After scanning three test sites and recording the elapsed scanning times (see Section 2.2), we observed that Skipfish took at most 28 minutes to scan a test website whereas Wapiti took at least an hour and a half to perform a full vulnerability scan. Similarly, the vulnerability assessments conducted using Arachni scanner consumed at least 23 minutes. Thus, Skipfish is considered the fastest scanner whereas Wapiti is the slowest one in detecting web-based vulnerabilities. Table 2 shows the scanning times for each evaluated scanner.

3.2. Crawler Coverage. We based our evaluation of the crawler coverage of the evaluated scanners on WIVET scores. The best crawling coverage percentage was $94 \%$ which was obtained by Arachni 1.0.2. The crawlers of the remaining three scanners covered at most $48 \%$ of the scanned web applications. As shown in Table 2, WIVET results show that Skipfish 2.1, Wapiti 2.3.0, and Arachni 0.4.3 covered 48\%, $44 \%$, and $19 \%$ of the crawled web applications, respectively.

By linking the crawling coverage with the speed of the evaluated scanners, note that the excellent crawling ability of 
TABLE 3: WAVSEP and Altoro Mutual: test cases detected by each scanner.

\begin{tabular}{lcccccccc}
\hline \multirow{2}{*}{ Scanner } & \multirow{2}{*}{ Version } & \multicolumn{4}{c}{ WAVSEP test cases } & \multicolumn{4}{c}{ IBM AppScan Test Site (Altoro Mutual) test cases } \\
& & SQL (135) & SQL FP (10) & XSS (73) & XSS FP (7) & SQL (13) & Blind SQL (1) & XSS (10) \\
\hline Arachni & 0.4 .3 & 134 & 2 & 47 & 0 & 9 & 1 & 8 \\
Arachni & 1.0 .2 & 135 & 0 & 64 & 0 & 2 & 0 & 4 \\
Wapiti & 2.3 .0 & 131 & 2 & 45 & 3 & 5 & 0 & 7 \\
Skipfish & 2.1 & 104 & 0 & 63 & 0 & 1 & 0 & 3 \\
\hline
\end{tabular}

TABLE 4: Vulnerability scanners' comparative assessment: extra features.

\begin{tabular}{lccccccc}
\hline Scanner & Version & $\begin{array}{c}\text { Web-based } \\
\text { Interface }\end{array}$ & Features & $\begin{array}{c}\text { Live } \\
\text { feedback }\end{array}$ & $\begin{array}{c}\text { Detailed } \\
\text { reports }\end{array}$ & $\begin{array}{c}\text { User } \\
\text { friendliness }\end{array}$ & Configuration \\
\hline Arachni & 0.4 .3 & Yes & More than 10 active and 20 passive checks & Yes & Yes & Easy & Flexible \\
Arachni & 1.0 .2 & Yes & More than 10 active and 20 passive checks & Yes & Yes & Easy & Flexible \\
Wapiti & 2.3 .0 & No & 10 modules for 9 vulnerability types & Yes & Yes & Easy & Basic \\
Skipfish & 2.1 & No & Different scanning modes and dictionaries & No & Yes & Easy & Basic \\
\hline
\end{tabular}

Arachni 1.0.2 could had negatively affected its speed. After comparing the crawler coverage of Arachni 0.4.3 and Arachni 1.0.2, we noted that the newer release was slower due to the significant improvement of its crawler. However, this does not necessarily imply that the advanced crawling capability of a given web vulnerability scanner is correlated with its poor performance. For example, although Skipfish was the fastest scanner, the results show that the coverage of its crawler was better than the coverage of both Wapiti 2.3.0 and Arachni 0.4.3.

3.3. Accuracy. We scanned IBM's AppScan test site (Altoro Mutual) to evaluate the level of accuracy of the scanning results reported by the chosen tools. We also used 225 of the test cases included in the Web Application Vulnerability Scanner Evaluation Project (WAVSEP) to clearly understand the accuracy weaknesses of the four evaluated scanners (see Table 3). Altoro Mutual site helped us to test our scanners against 13 SQL test cases, one blind SQL test case and ten XSS test cases. Similarly, we scanned the cases presented by WAVSEP benchmark which included 135 SQL true positive cases, 10 SQL false positive cases, 73 XSS true positive cases, and 7 XSS false positive cases. Based on WAVSEP benchmark, our results show that the four scanners were able to detect at least 104 SQL injection true positives and 45 XSS true positive cases. However, the majority of Altoro Mutual's SQL cases were not detected by Wapiti 2.0.3, Arachni 1.0.2, and Skipfish 2.1. Similarly, all the evaluated scanners were able to report at least $62 \%$ of WAVSEP's XSS true positive test cases whereas $50 \%$ of the evaluated scanners detected at most $40 \%$ Altoro Mutual XSS vulnerabilities. For WAVSEP's SQL and XSS false positive test cases, the four scanners reported at most $20 \%$ SQL cases and $43 \%$ XSS cases. Table 3 details the number of cases detected by each scanner and Section 5 discusses these observations.

3.4. Additional Features. To comprehensively evaluate the chosen web vulnerability scanners, we manually experimented with all the features offered by these tools and utilized the extra information documented in their corresponding websites. While we did not conduct a user study, we did not observe any notable problems with the graphical user interfaces of these scanners as we found them user friendly, easy to use, and understandable. Further, the tools provided live feedback which helped us to easily identify the vulnerable URLs and understand the types of detected vulnerabilities and their degrees of severity. Furthermore, we were satisfied with the vulnerability reporting features of these tools as all of them provided reports that included detailed statistics and explanations about the detected security vulnerabilities. Further, Arachni vulnerability assessment scanners offer extra features that allowed us to identify redundant URLs, classify the scanned URLs according to their security levels, and modify the crawling scope to include or exclude some web pages.

The four scanners varied in the types and number of security checks that can be performed. For example, Arachni 0.4.3 and Arachni 1.0.2 have many plugins that can be used for performing more than 30 active and passive vulnerability checks for a wide range of web-based vulnerabilities. Similarly, nine different types of web vulnerabilities can be checked using Wapiti 2.3.0 scanner including SQL and XSS injection attacks, file disclosure attacks, and XPath attacks. In addition, Skipfish has an extra feature that helps in improving the crawling coverage and including unlinked web pages in the vulnerability scanning process. This feature performs bruteforce based security checks and allows specifying the crawling scope as minimal, medium, or complete. Table 4 summarizes some of our scanners' additional features.

3.5. Quantitative Measures. Based on WAVSEP test cases, nine metrics were calculated to review the effectiveness of the four scanners in detecting SQL and XSS attacks. For each scanner, the true positive and true negative rates were measured to determine whether the results reported by the evaluated scanners truly reflect the security state of the scanned websites. We also measured the rates of false positives and false negatives reported by the scanners as well as 
TABLE 5: Scanners' detection accuracy of SQL attacks.

\begin{tabular}{|c|c|c|c|c|c|c|c|c|c|c|}
\hline Scanner & Version & TPR & TNR & FPR & FNR & PPV & NPV & FOR & Accuracy & $F$-measure \\
\hline Arachni & 0.4 .3 & $99.26 \%$ & $80 \%$ & $20 \%$ & $0.74 \%$ & $98.53 \%$ & $88.89 \%$ & $11.11 \%$ & $97.93 \%$ & $98.89 \%$ \\
\hline Arachni & 1.0 .2 & $100 \%$ & $100 \%$ & $0 \%$ & $0 \%$ & $100 \%$ & $100 \%$ & $0 \%$ & $100 \%$ & $100 \%$ \\
\hline Wapiti & 2.3 .0 & $97.04 \%$ & $80 \%$ & $20 \%$ & $2.96 \%$ & $98.50 \%$ & $66.67 \%$ & $33.33 \%$ & $95.86 \%$ & $97.76 \%$ \\
\hline Skipfish & 2.1 & $77.04 \%$ & $100 \%$ & $0 \%$ & $22.96 \%$ & $100 \%$ & $24.39 \%$ & $75.61 \%$ & $78.62 \%$ & $87.03 \%$ \\
\hline
\end{tabular}

TABLE 6: Scanners' detection accuracy of XSS attacks.

\begin{tabular}{|c|c|c|c|c|c|c|c|c|c|c|}
\hline Scanner & Version & TPR & TNR & FPR & FNR & PPV & NPV & FOR & Accuracy & $F$-measure \\
\hline Arachni & 0.4 .3 & $64.38 \%$ & $100 \%$ & $0 \%$ & $35.62 \%$ & $100 \%$ & $21.21 \%$ & $78.79 \%$ & $67.50 \%$ & $78.33 \%$ \\
\hline Arachni & 1.0 .2 & $87.67 \%$ & $100 \%$ & $0 \%$ & $12.33 \%$ & $100 \%$ & $43.75 \%$ & $56.25 \%$ & $88.75 \%$ & $93.44 \%$ \\
\hline Wapiti & 2.3.0 & $61.64 \%$ & $57.14 \%$ & $42.86 \%$ & $38.36 \%$ & $93.75 \%$ & $12.50 \%$ & $87.50 \%$ & $61.25 \%$ & $74.38 \%$ \\
\hline Skipfish & 2.1 & $86.30 \%$ & $100 \%$ & $0 \%$ & $13.70 \%$ & $100 \%$ & $41.18 \%$ & $58.82 \%$ & $87.50 \%$ & $92.65 \%$ \\
\hline
\end{tabular}

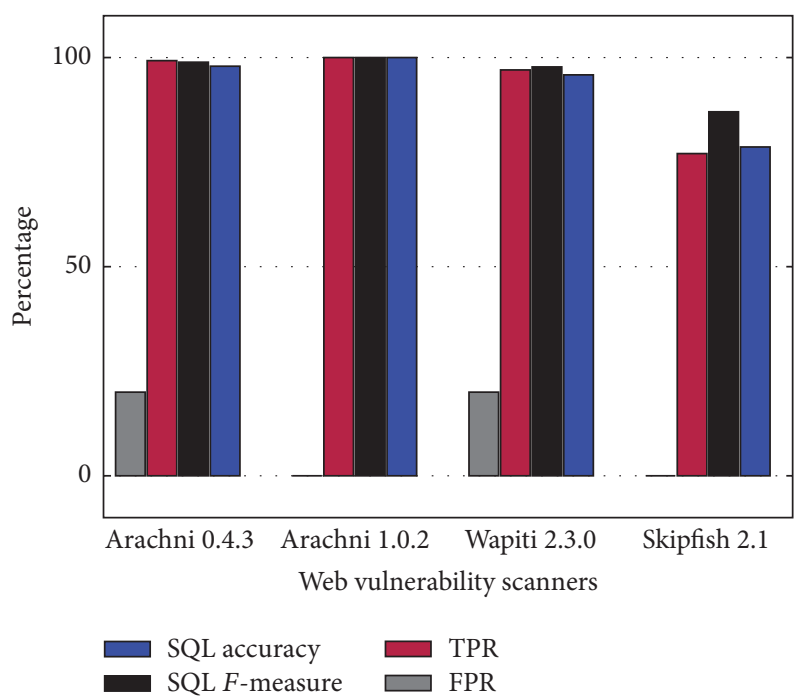

FIGURE 2: Scanners' performance: detection of SQL vulnerabilities.

the positive predictive and negative predictive values for each scanner. To clearly understand the detection accuracy of SQL and XSS attacks, the rate of true positives and true negatives to the total number of test cases and the $f$-measures were calculated for each scanner (see Section 2.2 and Table 1). The results of our analysis are presented in Tables 5 and 6 .

Based on the calculated $f$-measures, the detection accuracy of SQL vulnerabilities was over 97\% in Arachni 0.4.3 and Arachni 1.0.2 (see Figure 2). Although Skipfish's SQL detection accuracy was the lowest, it has a false positive rate of $0 \%$, a false negative rate of $23 \%$, and a true negative rate of $100 \%$. Furthermore, by considering the accuracy of each test which is measured through dividing the sum of true positives and true negatives by the total number of test cases, the rates of Arachni and Wapiti scanners were higher than $95 \%$. For SQL vulnerability detection, the accuracy measures of Arachni 1.0.2 were the best relative to the remaining three scanners. Further, Skipfish has a true positive rate of $77 \%$, which was the minimum observed TPR. For SQL attacks, the positive predictive values of all the scanners were very high ranging from $98.50 \%$ to $100 \%$. Also, the false negative rates of three of the evaluated scanners were less than 3\%. After ranking the four scanners according to their SQL vulnerability detection rates, Arachni 1.0.2 took the first position whereas Arachni 0.4.3, Wapiti 2.3.0, and Skipfish 2.1 took the second, third, and fourth positions, respectively.

The scanners' performance related to their effectiveness in detecting XSS vulnerabilities was also evaluated. Although Skipfish's accuracy rates for detecting SQL vulnerabilities were the lowest (see Figure 2), our results show that its detection rate for XSS vulnerabilities was very high in comparison with the values recorded for Wapiti 2.3.0 and Arachni 0.4.3. For all the evaluated scanners, we also recorded a false positive rate of $0 \%$ except for Wapiti which had a FPR of $42.86 \%$. Similarly, for all the four scanners except Wapiti, we also obtained true negative rates and positive predictive values of $100 \%$. The lowest true positive rates, $f$-measures, and accuracy values related to detecting XSS vulnerabilities were recorded for Wapiti 2.3.0 and Arachni 0.4.3 (see Figure 3). While the highest FNR related to SQL vulnerability detection was recorded for Skipfish (see Table 5), the number of XSS false negatives recorded for Skipfish was lower than the ones calculated for Arachni 0.4.3 and Wapiti 2.3.0. Similar to our observations for SQL vulnerability detection, the best accuracy measures related to the detection of XSS attacks were the ones recorded for Arachni 1.0.2. However, in contrast to our observations for SQL attacks, the results show that Skipfish 2.1 was more accurate than Wapiti 2.3.0 and Arachni 0.4.3 in detecting XSS vulnerabilities.

We observed that the newer version of Arachni was the most effective one in detecting SQL and XSS vulnerabilities. As presented in Tables 5 and 6, Arachni 1.0.2 has the highest true positive rate, true negative rate, accuracy value, and $f$ measure. In most of the cases, we did not note significant differences in performance among all the evaluated scanners. Generally, the overall performance of the four scanners was good as the lowest obtained $f$-measure was $74.38 \%$ and the highest FNR was $38.36 \%$. Further, the recorded true positive rates for most of the evaluated scanners are above 


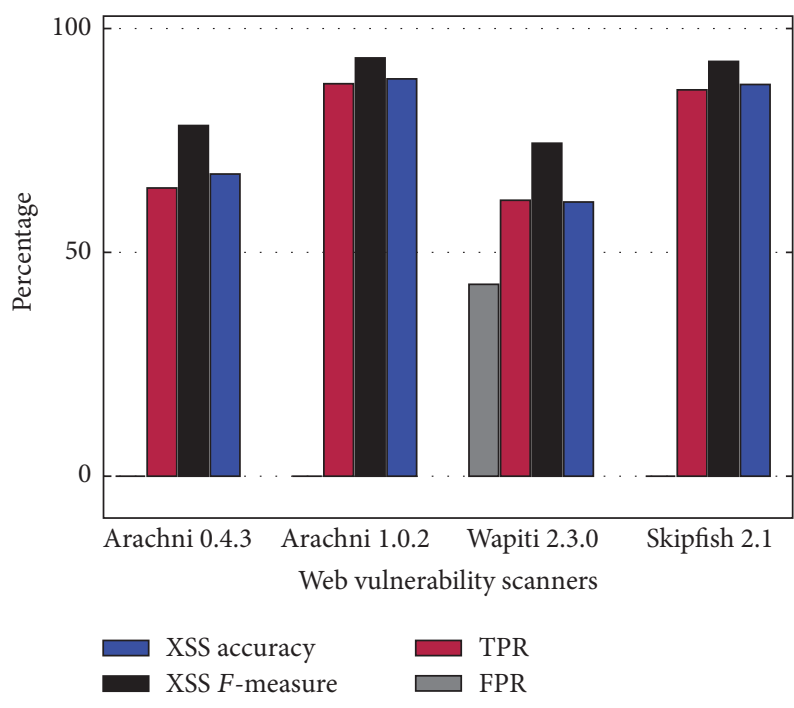

FIGURE 3: Scanners' performance: detection of XSS vulnerabilities.

$86 \%$ whereas the worst FPR is $42.86 \%$. Based on the results obtained after scanning IBM AppScan test site (see Table 3), the highest number of detected test cases was scored by Arachni. During our evaluation, scanning IBM AppScan using Arachni 1.0.2 unexpectedly stopped at the end of the experiment. The results shown in Table 3 are therefore according to the number of vulnerabilities detected before experiencing this technical problem. We also note that it was challenging to determine if the calculated false negative rates were actual false negatives or resulted from some crawler coverage issues.

\section{Case Study: Evaluating the Security State of Part of the Web Content}

We followed our performance evaluation of the scanners (see Section 3) with an evaluation of the overall security state of part of the web content as a case study. Two scanners were chosen for this purpose: Wapiti 2.3.0 and Arachni 0.4.3. First, we collected a sample of 140 unique URLs to popular webbased systems and used the chosen scanners to assess the security state of the websites in question (the collected URL dataset is available in [33]). Based on the quantitative analysis of the security scan reports generated by Wapiti 2.3.0 and Arachni 0.4 .3 , a security holistic view of part of the web content was then analyzed. In the following sections, we describe the conducted security scans, analyze the generated scan reports, and compare the results obtained from the two chosen scanners.

4.1. Dataset and Methodology. In this section, we describe the methodology followed to collect, scan, and analyze a sample of unique 140 URLs. Figure 4 summarizes the steps followed in our experiment. The following sections detail the decisions taken throughout each step in this case study and present the results of the conducted security vulnerabilities assessment.
4.1.1. Dataset Collection. One of the main objectives of this case study is to evaluate the overall security state of a part of the web content. The sample of the examined URLs includes different websites that vary in size, complexity, and topics. We used Google Trends [34] to select trending search terms from the period of 2004 to 2013. This was followed by performing Google searches on the trending keywords and using SEOquake [35] Firefox extension to harvest the first thirty URLs obtained from each search (the collected URL dataset is available in [33]). Figure 5 describes the followed steps to collect our dataset.

4.1.2. Methodology. To scan the collected URLs, two virtual machines were installed on Amazon EC2 [36] cloud-based service. The two instances were accessed using PuTTY [37] client and TightVNC [38] remote desktop controller. This was followed by installing Wapiti and Arachni web vulnerability scanners on the cloud instances and running the security vulnerabilities scans for each URL in our dataset. Note that Arachni was utilized to detect a wider range of security defects relative to Wapiti which was restricted to detecting SQL injection and XSS attacks.

We then analyzed and compared the results generated by each scanner to accurately understand the overall security state of part of the web content. Our analysis is based on the overall performance of each scanner as discussed in Section 3. For each website, we recorded the types of detected vulnerabilities by each scanner and their degree of severity. We were therefore able to identify the most common vulnerabilities in the examined URLs, rank them according to their degree of severity, and then obtain an idea about the general security state of the evaluated URLs.

4.2. Detected Vulnerabilities. The findings of the conducted experiments and the level of agreement between the web vulnerability scanners evaluated in this study are discussed in this section.

4.2.1. Arachni. After scanning 140 unique URLs using Arachni, we found that $98 \%$ of them had security vulnerabilities. $60 \%$ of the vulnerable web servers included high-severity vulnerabilities and $96 \%$ had informational vulnerabilities. Arachni reports also show that $72 \%$ of the vulnerable web servers included low-severity vulnerabilities and $65 \%$ had medium-severity vulnerabilities.

Based on Arachni results, our sample had seven highseverity, four medium-severity, three low-severity, and three informational web vulnerability types. Table 7 summarizes the types, numbers, and severity ratings of the web vulnerabilities that were detected by Arachni and ranks them according to their frequency of occurrence. According to Arachni, around $64 \%$ of the web server with high-severity vulnerabilities were also vulnerable to cross-site request forgery (CSRF) attack. Further, cross-site scripting vulnerabilities (XSS) were the second most frequently observed security issues in our sample (36\% of the scanned URLs that had high-severity vulnerabilities). Arachni also reported that $15 \%$ of the sample were vulnerable to SQL injection attacks. Also, $4 \%$ of the evaluated websites had backdoor files and file inclusion 


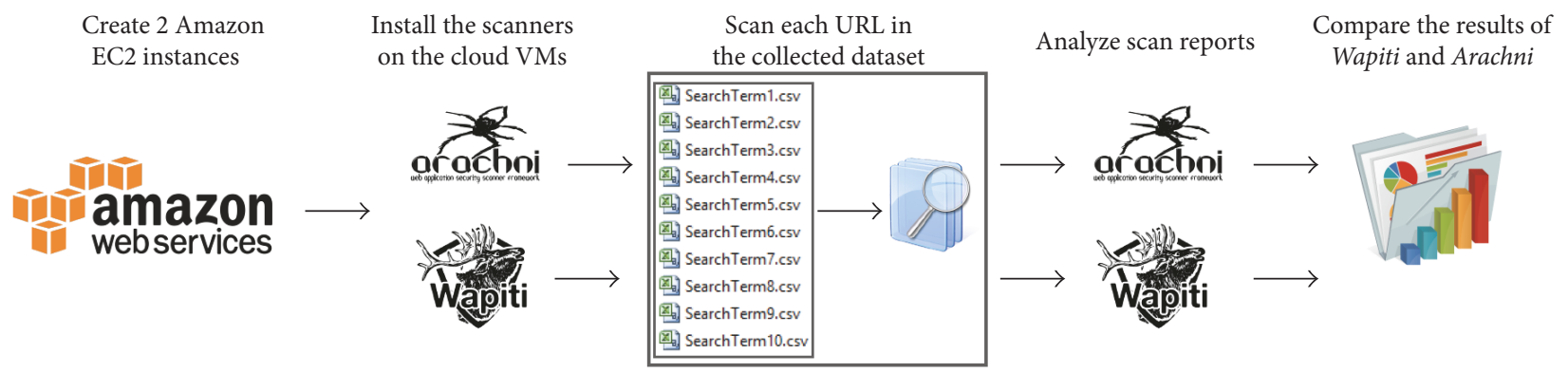

FIGURE 4: Arabic websites security evaluation: the followed approach.

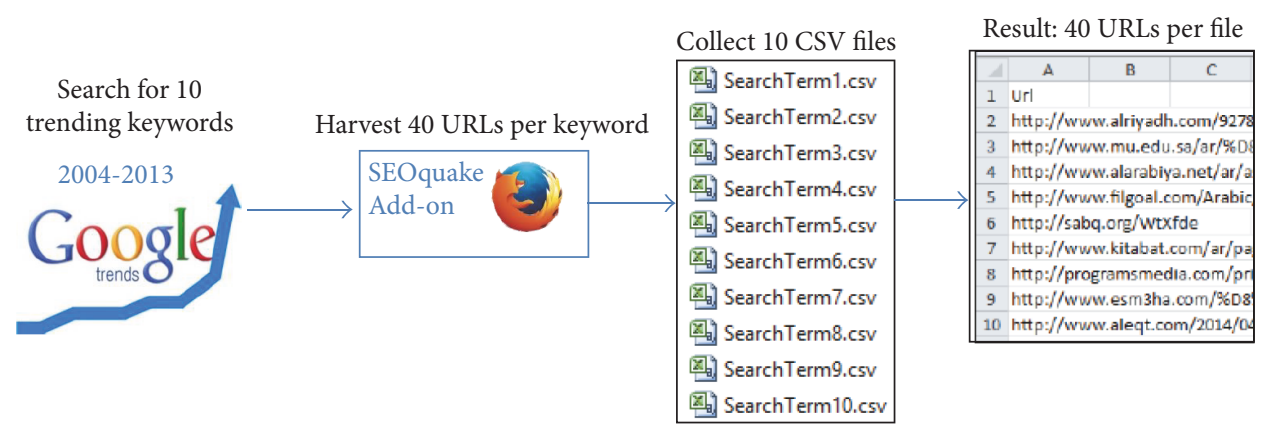

FIgURE 5: Collecting the sample URLs.

TABLE 7: Arachni’s reported vulnerabilities: a summary.

\begin{tabular}{lcc}
\hline Severity & Vulnerability & \# of vulnerable URLs \\
\hline \multirow{4}{*}{ High } & CSRF & 53 \\
& XSS & 30 \\
& SQL & 21 \\
& Source code Disclosure & 6 \\
& Backdoor File & 5 \\
& File Inclusion & 5 \\
Medium & X-Forwarded-For & 1 \\
\hline \multirow{4}{*}{ Low } & Common directory & 68 \\
& Unencrypted passwords & 43 \\
& Backup files & 9 \\
Informational & Unvalidated redirect & 2 \\
\cline { 2 - 3 } & Common sensitive files & 77 \\
& Password autocomplete & 42 \\
& Directory listing & 4 \\
\hline \multirow{4}{*}{ Interesting response } & 123 \\
& Insecure cookie & 90 \\
& Email disclosure & 45
\end{tabular}

vulnerabilities. Source code disclosure vulnerabilities were also observed with a percentage of $7 \%$ of our URLs that had high-severity vulnerabilities. The most spread high-severity vulnerabilities in the evaluated web-based applications were CSRF, XSS, and SQL injection attacks.

As presented in Table 7, 90 of the scanned web servers were vulnerable to four types of medium-severity vulnerabilities. Among medium-severe vulnerabilities, at least $75 \%$ were vulnerable to the attacks related to the use of common directories on web servers. Arachni's results also showed that at least $30 \%$ of our evaluated websites did not implement proper encryption mechanisms (e.g., users' credentials were sent without encryption). Arachni also reported that $6 \%$ had stored their backup files in the same web servers. Furthermore, unvalidated redirect security defects were observed in $2 \%$ of the scanned URLs with medium-severity vulnerabilities. In short, $65 \%$ of the evaluated websites had medium-severity vulnerabilities that could have been caused by incorrect software development practices.

Three types of low-severity web vulnerabilities were detected by Arachni in at least $71 \%$ of the evaluated URLs. In at least $77 \%$ of these vulnerable web servers, Arachni was able to locate some files (e.g., robot.txt, sitemap.xml, and config.php) that might reveal sensitive information about the web servers in question. For instance, attackers might use such files to view the names of some hidden directories or disclose information about the configurations of the respective servers. In $30 \%$ of the sample, Arachni was also able to find some form fields with password autocompletion option enabled. Similarly, Arachni reports show that a directory listing option was permitted in $4 \%$ of the URLs that included low-severity vulnerabilities.

Informational vulnerabilities were found in $95 \%$ of our sample in which more than $92 \%$ were related to receiving "Not Found" error message (i.e., 404 HTTP responses) from the requested web servers. Arachni also reported that over $67 \%$ of the evaluated URLs had informational vulnerabilities 
TABLE 8: Arachni: highest number of occurrences of each vulnerability type.

\begin{tabular}{lcc}
\hline Vulnerability & Severity & Highest number \\
\hline Common directory & Medium & 54 \\
XSS & High & 33 \\
SQL & High & 29 \\
Backup file & Medium & 27 \\
CSRF & High & 26 \\
PW autocomplete & Low & 25 \\
Interesting response & Informational & 25 \\
Backdoor file & High & 24 \\
Source code disclosure & High & 18 \\
File inclusion & High & 10 \\
Common sensitive files & Low & 10 \\
Email disclosure & Informational & 8 \\
Insecure cookie & Informational & 7 \\
Unencrypted password & Medium & 5 \\
Directory listing & Low & 1 \\
X-Forwarded-For & High & 1 \\
\hline
\end{tabular}

related to sending data using unencrypted cookies. In $32 \%$ of the collected URLs, Arachni was also able to discover some email disclosure vulnerabilities in which some email addresses that were written in some scripts or code comments were accessible by the scanner.

This experiment enabled us to identify the most spread web vulnerabilities and rank them accordingly. Without considering the severity degrees of the detected vulnerabilities, our results show that the average number of web vulnerabilities found in one web server was 21 . For each web vulnerability type, we recorded the highest number of vulnerabilities found in one web server. For example, the highest number of SQL injection, XSS, and CSRF vulnerabilities found in a website was 29,33 , and 26 , respectively. For each vulnerability type, Table 8 ranks the number of web vulnerabilities detected in one web server based on their frequency of occurrence.

4.2.2. Wapiti. We used Wapiti to detect two types of web vulnerabilities: SQL injection and XSS attacks (see Figure 7 for a sample scan result). Based on Wapiti results, 86 out of our collected 140 URLs had either SQL injection or XSS vulnerabilities. Around $92 \%$ of the 86 vulnerable web servers had high-severity SQL injection or XSS vulnerabilities. Wapiti also reported that at least $19 \%$ of our collected sample was vulnerable to low-severity risks. Wapiti's scan results also indicated that at least $81 \%$ of the URLs that had high-severity vulnerabilities were vulnerable to SQL injection attacks. Similarly, Wapiti detected high-severity cross-side scripting vulnerabilities in $28 \%$ of the evaluated websites. The highest numbers of SQL injection and cross-side scripting vulnerabilities discovered in one web-based application were 600 and 234, respectively. Additionally, Wapiti found that the highest number of HTTP internal server error responses received from one web server is 759 . Table 9 summarizes the number of URLs that were reported as vulnerable to
TABLE 9: Wapiti: detection of SQL and XSS vulnerabilities.

\begin{tabular}{lcc}
\hline Vulnerability & Number of vulnerable URLs & Highest number \\
\hline XSS & 64 & 600 \\
SQL & 39 & 234 \\
\hline
\end{tabular}
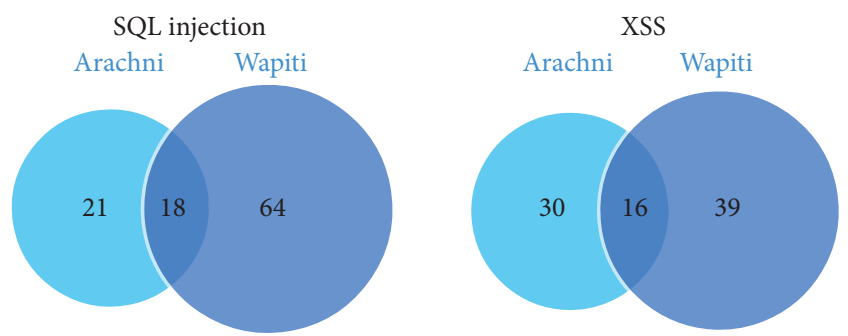

FIGURE 6: Wapiti versus Arachni: detection of vulnerable URLs.

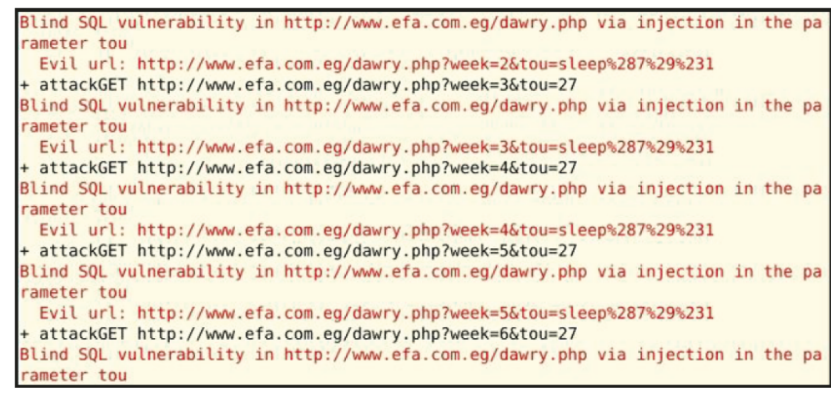

FIGURE 7: Wapiti web vulnerability scanner: sample scan result.

high-severity XSS or SQL attacks as well as the highest numbers of SQL or XSS vulnerabilities that were found in one web server.

4.2.3. Arachni and Wapiti: A Comparison. After scanning the same sample using Arachni and Wapiti, we compared their results and quantified the number of URLs that were reported by the two scanners as vulnerable to SQL injection or XSS attacks. Based on the reports generated by Arachni, $15 \%$ of our collected sample was vulnerable to SQL injection attacks. However, the number of URLs that were reported as vulnerable to SQL injection attacks by Wapiti scanner was at least two times higher than Arachni's one. According to Wapiti, around $46 \%$ had SQL injection vulnerabilities. Both scanners indicated that $12.86 \%$ of the evaluated sample was vulnerable to SQL injection attacks. On the other hand, the findings of our comparison also showed that $11.43 \%$ of the scanned URLs were reported as vulnerable to cross-side scripting attacks by both scanners. For XSS vulnerabilities, there was a little difference between those reported positive by the two scanners (i.e., Wapiti reported $27.86 \%$ relative to 21.43\% reported by Arachni's). Both Wapiti and Arachni indicated that at least $12.86 \%$ were vulnerable to SQL injection attacks whereas $11.43 \%$ had XSS vulnerabilities (see Figure 6). 


\section{Further Discussion}

We combined the results of our first experiment, which compared the performances of four scanners based on objective measures, with the findings of the second experiment which evaluated the performance of two scanners on a large set of selected URLs. Before conducting our case study and based on the results obtained from our scanners' comparative evaluation, we expected Arachni to report less number of security vulnerabilities compared to Wapiti, as the crawler coverage of Arachni was 19\% whereas Wapiti's crawler coverage was $44 \%$. In our case study, we observed that the number of SQL and XSS vulnerabilities reported by Wapiti was higher than the results reported by Arachni. As shown in Table 7 , Arachni's reports showed that $15 \%$ of our sample was vulnerable to high-severity SQL attacks and $21 \%$ of the collected URLs were vulnerable to high-severity XSS attacks. According to Wapiti, around $46 \%$ of the URLs examined in our case study were vulnerable to SQL attacks and XSS vulnerabilities existed in $28 \%$ of our sample. However, this might not necessarily correlate with Wapiti's crawler coverage capabilities as our observations also showed that Wapiti had the highest FPR for XSS and SQL attacks (as shown in Tables 5 and 6). Additionally, the results showed that Arachni's vulnerability detection rates were higher than Wapiti's ones. Hence, because Arachni's crawler coverage was the lowest, there is a possibility that many web pages were not reached by Arachni's crawler.

Although there were little differences in $f$-measures and detection accuracy rates for SQL and XSS vulnerabilities between Wapiti and Arachni, we observed that Wapiti had higher false negative rates and lower true negative rates for both SQL and XSS attacks (see Tables 5 and 6). Thus, although Wapiti's crawler coverage rate was higher than Arachni's one, there is a possibility that Wapiti had not detected some vulnerable URLs that were examined in the conducted case study. For XSS attacks, we also observed that Wapiti 2.3.0 and Arachni 0.4.3 had the lowest true positive rates compared to Skipfish 2.1 and Arachni 1.0.2. The percentage of URLs that were vulnerable to XSS attacks might therefore be higher than those reported by the two scanners included in our case study. This finding might also be supported by the number of WAVSEP XSS true positive test cases that were detected by Arachni 0.4.3 and Wapiti 2.3.0 (see Table 3). Based on the comparative evaluation, both scanners had detected at most 47 XSS cases out of WAVSEP's 73 true positive XSS test cases. Further, although both scanners had high true positive rates for SQL vulnerabilities, Wapiti had detected only 5 out of 13 Altoro Mutual's SQL true positive cases whereas the number of cases detected by Arachni 0.4.3 was 9 (see Table 3). Therefore, the number of URLs that were vulnerable to SQL attacks might also be higher than the numbers reported by Wapiti and Arachni.

We observed notable differences between the scan results of Wapiti and Arachni. Although Arachni's reports showed that 21 of the examined web servers had SQL vulnerabilities, Wapiti's scan results indicated that 64 of our 140 URLs were vulnerable to SQL attacks. However, only 18 URLs were reported as vulnerable to SQL attacks by the two scanners. For
XSS vulnerabilities, we note that the two scanners indicated that 16 web servers were vulnerable although each scanner flagged at least 30 URLs as vulnerable to XSS attacks (see Figure 6). Although the results of our scanners' comparative assessment did not reveal notable differences between the performances of the four evaluated scanners, there was a high level of disagreement between the scan results of Wapiti and Arachni. This suggests that web vulnerability detection scanners have limitations that might be related to their crawling capabilities, detection accuracy, or other implementation details that need to be researched. Our observations also show that the slow performance of the studied scanners might not necessarily imply that the respective scanner has a good crawling coverage. We observed that Wapiti's long scanning time resulted from scanning web pages containing similar or duplicate content. This means that the there was a considerable increase in the scanning time that resulted from sending a high number of unnecessary HTTP requests and repeatedly filling many form elements without guaranteeing the coverage of all web pages belonging to the websites in question.

By observing the highest numbers of XSS and SQL injection vulnerabilities that existed in one website, we also note variations between the scan results reported by Arachni and Wapiti. Although Arachni's reports showed that the highest number of SQL injection vulnerabilities that were found in one website was 29 (as shown in Table 8), Wapiti's scan results indicated that 600 SQL vulnerabilities were detected in one website. Similarly, Wapiti found 234 XSS vulnerabilities in one website whereas the highest number of XSS vulnerabilities detected in one website by Arachni was 33. There are therefore considerable differences between the scan results of Wapiti and Arachni although our quantitative comparative performance assessment did not reveal notable differences between the detection accuracy rates of these scanners. This variation can be explained either by the crawler coverage which is presented in Table 2 or by the false alarm rates of Wapiti and Arachni.

Our comparative assessment results showed that Wapiti's observed false alarm rate for XSS vulnerabilities was $42.86 \%$ whereas no false positives were recorded for Arachni 0.4.3 (see Table 6). For SQL injection vulnerabilities, our observations also showed that a false positive rate of $20 \%$ was observed for the two scanners; however, our case study showed that there was a notable difference between the numbers of SQL vulnerabilities detected by the two scanners. Because Arachni's crawler coverage percentage was poor, there is a possibility that Arachni's crawler did not scan all the pages that were scanned by Wapiti. However, this does not imply that Wapiti's results were more accurate since we observed that Wapiti had repeatedly scanned a considerable number of URLs containing similar web content.

\section{Guidelines and Recommendations}

Our experimental findings highlighted limitations related to scanners' crawling capabilities, reporting and visualization features, efficiency, and vulnerability detection accuracy. In this section, we note some areas that should be addressed by developers in order to increase the effectiveness of their tools. 
We recommend system designers to utilize the available security benchmarks and test sites (e.g., the ones shown in Figure 1) to evaluate the performance of their open source vulnerability detection solutions. This could contribute to improving the capabilities of open source scanners as it would allow comparing the quantities and types of vulnerabilities that were detected by open source scanners with those discovered by well-known proprietary scanners (e.g., IBM Security AppScan [8]). We also recommend tool developers to improve the crawling capabilities of their tools by ensuring that all the clickable elements in a crawled web page are identified and thus all the web pages that belong to the examined domains are traversed. For increasing the coverage of attack classes checked by web vulnerability scanners, tool developers could also enhance the comprehensiveness of their tests by validating all possible combinations of inputs to web forms while ensuring that the efficiency of their tools is not compromised. The elimination of duplicate links before starting the vulnerability scanning process is also recommended for improving the efficiency of vulnerability detection.

Designers of open source web vulnerability scanners are also recommended to improve the accuracy of the security verification checks incorporated in their tools to simplify the task of manually checking whether the vulnerabilities reported by these scanners are false positives or could actually be exploited by attackers [39]. That is, reporting web pages as vulnerable to exploitable attacks when in fact they do not exist increases the effort spent by penetration testers on validating the accuracy of the results reported by these tools. For instance, web vulnerability scanners might sometimes report security vulnerabilities related to having cookies that are not flagged as secure when in fact they store nonsensitive data (e.g., user preferences) that is of no value to attackers. Platform designers could therefore address this problem by identifying the characteristics of nonsensitive cookies to avoid reporting them as potential security vulnerabilities. Web vulnerability scanners might also sometimes report vulnerabilities relating to locating server files without verifying whether the content of these files could actually disclose hidden information about the configurations of the web servers in question or not. We also recommend improving the reporting mechanisms implemented in open source scanners by presenting the user with more details about detected vulnerabilities and highlighting potential false positives. Reports generated by penetration testing tools should also include more guidance to draw the testers' attention to the factors that could characterize false positives and provide remediation advice on how to address detected vulnerabilities. Penetration testers should also be made aware of the circumstances that could cause a scanner to not detect certain vulnerabilities or misinterpret the observed behavior of the scanned website and report a vulnerability that does not exist.

From a usability viewpoint, tool designers are recommended to implement web-based interfaces of their open source scanners to allow web developers to access these scanners and run their scans easily. They are also recommended to improve the vulnerability reporting and visualization features of these tools to ensure that users are able to monitor the scanning processes in real time. Users should also be provided with various options allowing them to customize and control the scope of their scans [40].

With the emergence of new client and server side technologies, the developers of open source vulnerability scanners should continuously update their solutions to provide support to a variety of web platforms and protocols. A significant attention should be given to resolving the compatibility and interoperability issues that prevent software developers from scanning their web-based systems. Although the inclusion of all attack classes in one database is challenging, tool developers should ensure that their vulnerability databases are regularly updated to include newly discovered attacks. In order for a vulnerability scanning process to be successful and comprehensive, all applications states should also be thoroughly examined to ensure that all complex attack scenarios are handled by the corresponding web vulnerability scanner [5].

\section{Related Work}

Prior work addressed web security vulnerabilities by either researching the ways to improve dynamic testing techniques [41], proposing or evaluating static analysis testing mechanisms [42-45], or using these testing approaches to evaluate the security of web-based systems [46-49]. The majority of research studies that evaluated the performance of web vulnerability scanners suggested that they have limited crawling capabilities and high false positive rates. The findings reported in this paper show that there is a low level of agreement between the results reported by different open source web vulnerability scanners. In the following subsection, we summarize the literature related dynamic testing tools and the detection of web vulnerabilities.

7.1. Web Security Scanners: Detection Effectiveness. Evaluating the security of web-based applications and detecting the security vulnerabilities in these multisourced systems is a complex problem that can be addressed in many different ways [5052]. With the increasing complexity of web-based systems and the emergence of new development technologies that vary in their support for security, the demand for tools that automatically detect security vulnerabilities is growing [5355]. Penetration testing is one of the widely used techniques that is applied to detect security vulnerabilities in a costeffective way and without requiring technical expertise $[6,53$, $56,57]$.

In spite of the advantages of tool-based web penetration testing approaches, previous research attempts proved that web vulnerability scanners have poor performance and found that they are often unable to detect many security vulnerabilities $[6,53,58,59]$. The limited discoverability capability is also one of the drawbacks of web vulnerability scanners (i.e., security vulnerability cannot be detected until the crawler of the corresponding scanner reaches the URL in question) [5]. Although the results generated by these scanners are mostly regarded by software developers as comprehensive and accurate [56], prior work found that these scanners only detect a subset of the security vulnerabilities in the evaluated systems and give a significant number of false alarms $[53,56]$. 
Prior work has also addressed the performance of web security vulnerability scanners by either evaluating the detection effectiveness of a set of scanners $[18,19,32,58]$ or developing techniques that can be incorporated into these tools to increase their detection accuracy [5, 60, 61]. Other research attempts focused on comparing the effectiveness of dynamic testing with other security testing approaches (e.g., static testing or manual code review) [62]. Vieira et al. used some commercial web vulnerability detection tools for quantifying the number of security vulnerabilities in publicly accessible web services and observed a high false positive rate [17]. Consistent with our findings, the experimental results presented in [17] highlight limitations in the vulnerability detection and crawling capabilities of dynamic testing tools and show that there are major differences between the types and quantities of security vulnerabilities detected by different scanners.

Their findings also suggested that there are variations between the results reported by different web vulnerability scanners. Similarly, three commercial web vulnerability detection scanners were evaluated in [18] by injecting SQL and XSS vulnerabilities into web-based systems and testing the scanners' effectiveness in detecting these security flaws. Their results furthermore suggest that these scanners leave some vulnerabilities undetected and produce inconsistent results. Although the observations reported in $[17,18]$ show that the percentages of false positives were high, the FPRs of $75 \%$ of the evaluated scanners in this study were less than or equal to $20 \%$. This implies that the inconsistencies between the scan results of different scanners might not necessarily be linked to their poor performance.

Doupé et al. [32] also highlighted the limitations in the crawling capabilities of web vulnerability scanners after evaluating eight commercial and three open source scanners. Although some open source scanners were included in the evaluation conducted in [32], their comparative assessment is based on scanning one web-based system that was specifically developed for the purpose of their study. In our study, we evaluate the selected scanners based on a systematic approach that considers scanning a dataset that includes more than 140 distinct web-based systems. Clearly, there is a lack of studies that thoroughly analyze the effectiveness of open source web vulnerability scanners based on well-known security benchmarks and standards.

A number of researchers have focused on identifying the root causes of web vulnerabilities and proposed solutions to mitigate the effects of web security attacks [63-66]. For instance, as an attempt to explore the correlations between security vulnerabilities and software faults, Fonseca and Vieira analyzed a large collection of security patches and found that the majority of SQL injection and XSS attacks were not caused by software bugs [63]. Soska and Christin addressed the maliciousness of web content in a different way by implementing a classification system that predicts whether a given web server will be compromised in the future [64]. In this study, we focused on comparing the effectiveness of two open source web vulnerability scanners in detecting SQL injection and XSS attacks. Our results show that there is a high level of disagreement between the results reported by the two scanners which implies that there is a demand for improving the efficiency, effectiveness, and detection accuracy of these scanners.

\section{Limitations and Future Work}

While our web vulnerability scanners' comparative evaluation included three scanners that are among the widely used open source web vulnerabilities detection tools, our results might not be generalized to all other similar open source tools. However, our evaluation approach can be applied to any set of open source or commercial web vulnerability scanners. Although the dataset collected for our case study included 140 URLs only, the approach followed to collect, scan, analyze, and evaluate the security state of these web applications can be applied to other web-based systems as well. The sample examined in our case study had only 140 URLs which were collected based on a predefined approach that allowed us to collect a sample of websites that vary in their size, complexity, and development technologies. Our case study can therefore be repeated to include a larger URL dataset. In our case study, we compared the results of the evaluated scanners based on their detection of SQL and XSS vulnerabilities only. Future work might consider other types of web vulnerabilities (e.g., CSRF and source code disclosure). Future research efforts might also utilize the methodology followed in this paper to evaluate commercial web vulnerability scanners. Other research efforts might also be directed toward investigating the level of agreement between open source and commercial web vulnerability scanners. Our experiment could also be applied on a large set of open source web vulnerability scanners to further understand the factors that might contribute to improving the performance of these scanners. In future work, special attention should also be given to thoroughly investigating the effect of false positives on the quantities and types of security vulnerabilities reported by the scanners. For example, repeating the tests multiple times could reveal inconsistencies in the scanners' reports and thus help researchers in drawing more accurate conclusions on the detection accuracy of the scanners.

\section{Concluding Remarks}

The widespread adoption of web vulnerability scanners and the differences in the functionality provided by these tool-based vulnerability detection approaches increase the demand for testing their detection effectiveness. Although there are many previously conducted research studies that addressed the performance characteristics of web vulnerability detection tools by either quantifying the number of false alarms or measuring the corresponding crawler coverage, the scope of the majority of these studies is limited to commercial tools.

Despite the advantages of dynamic testing approaches in detecting software vulnerabilities at any stage in the software development process, the literature lacks studies that comprehensively and systematically evaluate the performance of open source web vulnerability scanners based on sound measures. The main objectives of this study are 
to assess the performance of open source scanners from multiple perspectives and to examine whether the costeffectiveness of these tools negatively correlates with their detection capability. We expect the results of this research work to guide tool developers in enhancing the software processes followed while designing these tools, which in turn is expected to encourage software engineers to effectively utilize web vulnerability detection scanners during and after releasing their software products.

The results of our comparative evaluation of a set of open source scanners highlighted variations in the effectiveness of security vulnerability detection and indicated that there are correlations between different performance properties of these scanners (e.g., scanning speed, crawler coverage, and number of detected vulnerabilities). There was a considerable variance on both types and numbers of detected web vulnerabilities among the examined tools.

We followed our comparative assessment with a case study in which we evaluate the level of agreement between the results reported by two open source web vulnerability scanners. These scanners were utilized to examine the web security vulnerabilities in 140 URLs and quantify the number of low, medium, and high degree security vulnerabilities that existed in the evaluated URLs. By considering the fact that the examined scanners indicated that only 18 URLs were vulnerable to SQL injection attacks, this might suggest that the inconsistencies between the scan results of different tools might had been caused by factors other than performance differences. This finding might also be supported by the fact that although our scanners' comparative assessment did not show significant performance differences among the evaluated scanners, we observed a high level of disagreement between the scan results. Therefore, additional research should be directed toward identifying the factors that lead different widely known software verification tools to report inconsistent results.

\section{Conflicts of Interest}

The authors declare that there are no conflicts of interest regarding the publication of this paper.

\section{Acknowledgments}

This project was supported by NSTIP Strategic Technologies Program (no. 11-INF1855-02) in the Kingdom of Saudi Arabia. The authors thank Monirah Alshreef for preparing the URL dataset and conducting the experiments reported in the present study. This work was supported in part by KACST.

\section{References}

[1] "OWASP: the ten most critical web application security risks," The Open Web Application Security Project, 2013.

[2] "Internet security threat report," Tech. Rep., Symantec, 2015.

[3] M. Garnaeva, V. Chebyshev, D. Makrushin, and A. Ivanov, "IT threat evolution in Q1 2015," Tech. Rep., Kaspersky, 2015.

[4] Gartner, "The next three years in security threats," 2015 http:// www.gartner.com/smarterwithgartner/the-next-three-years-insecurity-threats/.
[5] A. Doupé, L. Cavedon, C. Kruegel, and G. Vigna, "Enemy of the state: a state-aware black-box web vulnerability scanner," in Proceedings of the USENIX Security Symposium, pp. 523-538, 2012.

[6] N. Antunes and M. Vieira, "Comparing the effectiveness of penetration testing and static code analysis on the detection of SQL injection vulnerabilities in web services," in Proceedings of the 15th IEEE Pacific Rim International Symposium on Dependable Computing (PRDC '09), pp. 301-306, November 2009.

[7] F. Elberzhager, J. Münch, and V. T. N. Nha, "A systematic mapping study on the combination of static and dynamic quality assurance techniques," Information and Software Technology, vol. 54, no. 1, pp. 1-15, 2012.

[8] IBM AppScan, 2015 http://www-03.ibm.com/software/products/ en/appscan.

[9] HP Web Inspect, 2015 http://www8.hp.com/us/en/software-solutions/webinspect-dynamic-analysis-dast/.

[10] Acunetix Web Vulnerability Scanner, 2015 http://www.acunetix .com/vulnerability-scanner/.

[11] K. McQuade, "Open source web vulnerability scanners: the cost effective choice?" in Proceedings of the Conference for Information Systems Applied Research, vol. 2167, p. 1508, 2014.

[12] S. Chen, "SECTOOL Market: price and feature comparison of web application scanners," 2015 http://goo.gl/ZUKaK8.

[13] D. Geer, "Are companies actually using secure development life cycles?" Computer, vol. 43, no. 6, pp. 12-16, 2010.

[14] J. Witschey, "Secure development tool adoption in opensource," in Proceedings of the 2013 Companion Publication for Conference on Systems, Programming, \& Applications: Software for Humanity, pp. 105-106, ACM, 2013.

[15] G. A. Di Lucca and A. R. Fasolino, "Testing Web-based applications: The state of the art and future trends," Information and Software Technology, vol. 48, no. 12, pp. 1172-1186, 2006.

[16] E. Fong and V. Okun, "Web application scanners: definitions and functions," in Proceedings of the 40th Annual Hawaii International Conference on System Sciences (HICSS'07), January 2007.

[17] M. Vieira, N. Antunes, and H. Madeira, "Using web security scanners to detect vulnerabilities in web services," in Proceedings of the IEEE/IFIP International Conference on Dependable Systems \& Networks, pp. 566-571, IEEE, 2009.

[18] J. Fonseca, M. Vieira, and H. Madeira, “Testing and comparing web vulnerability scanning tools for SQL injection and XSS attacks," in Proceedings of the 13th Pacific Rim International Symposium on Dependable Computing, pp. 365-372, IEEE, 2007.

[19] L. Suto, Analyzing the effectiveness and coverage of web application security scanners. San Francisco, October, 2007.

[20] Skipfish scanner, 2012 https://code.google.com/p/skipfish/.

[21] Arachni Web Application Security Scanner Fremework, 2015 http://www.arachni-scanner.com/.

[22] The Web Application Vulnerability Scanners Evaluation Project, 2013 https://code.google.com/p/wavsep/.

[23] Wapiti- web application vulnerability scanner, $2014 \mathrm{http}: / /$ wapiti .sourceforge.net/.

[24] Vega Vulnerability Scanner, 2014 https://subgraph.com/vega/.

[25] w3af: Open Source Web Application Security Scanner, 2013 http://w3af.org/.

[26] IronWASP, 2014 https://ironwasp.org/.

[27] Web Scanner Test Site, 2015 http://webscantest.com/.

[28] Test Website for Acunetix Web Vulnerability Scanner, 2005 http://testaspnet.vulnweb.com/. 
[29] AltoroMutual, 2015 http://demo.testfire.net/.

[30] Web Input Vector Extractor Teaser, 2014 https://github.com/ bedirhan/wivet.

[31] N. Antunes and M. Vieira, "Benchmarking vulnerability detection tools for web services," in Proceedings of the IEEE 8th International Conference on Web Services (ICWS '10), pp. 203-210, IEEE, July 2010.

[32] A. Doupé, M. Cova, and G. Vigna, "Why Johnny can’t pentest: an analysis of black-box web vulnerability scanners," in Detection of Intrusions and Malware, and Vulnerability Assessment, vol. 6201, pp. 111-131, Springer, 2010.

[33] Performance-Based Comparative Assessment of Open Source Web Vulnerability Scanners List of Scanned Websites, 2016 https://goo.gl/XjlFMl.

[34] GoogleTrends, 2015 https://www.google.com/trends/.

[35] SEOquake Firefox Extension, 2012 http://www.seoquake.com/.

[36] Amazon Elastic Compute Cloud, 2015 http://aws.amazon.com/ ec2/.

[37] PuTTY, 2015 http://www.putty.org/.

[38] TightVNC Software, 2014 http://www.tightvnc.com/.

[39] A. Austin and L. Williams, "One technique is not enough: a comparison of vulnerability discovery techniques," in Proceedings of the International Symposium on Empirical Software Engineering and Measurement (ESEM '11), pp. 97-106, IEEE, 2011.

[40] H.-Z. Shi, B. Chen, and L. Yu, "Analysis of web security comprehensive evaluation tools," in Proceedings of the 2nd International Conference on Networks Security, Wireless Communications and Trusted Computing (NSWCTC '10), pp. 285-289, April 2010.

[41] N. F. Awang and A. A. Manaf, "Detecting Vulnerabilities in Web Applications Using Automated Black Box and Manual Penetration Testing," in Advances in Security of Information and Communication Networks, vol. 381, pp. 230-239, Springer, 2013.

[42] V. B. Livshits and M. S. Lam, "Finding security vulnerabilities in java applications with static analysis," in Proceedings of the 14th Usenix Security Symposium, pp. 271-286, 2005.

[43] Y. Xie and A. Aiken, "Static detection of security vulnerabilities in scripting languages," in Proceedings of the USENIX Security Symposium, vol. 6, pp. 179-192, 2006.

[44] K. Goseva-Popstojanova and A. Perhinschi, "On the capability of static code analysis to detect security vulnerabilities," Information and Software Technology, vol. 68, pp. 18-33, 2015.

[45] G. Díaz and J. R. Bermejo, "Static analysis of source code security: assessment of tools against SAMATE tests," Information and Software Technology, vol. 55, no. 8, pp. 1462-1476, 2013.

[46] A. Alarifi, M. Alsaleh, and A. M. Al-Salman, "Security analysis of top visited Arabic Web sites," in Proceedings of the 15th International Conference on Advanced Communication Technology (ICACT '13), pp. 173-178, IEEE, 2013.

[47] M. Alsaleh and A. Alarifi, "Analysis of web spam for non-english content: toward more effective language-based classifiers," PLOS ONE, vol. 11, no. 11, Article ID e0164383, 2016.

[48] A. Alarifi and M. Alsaleh, "Web spam: a study of the page language effect on the spam detection features," in Proceedings of the 11th IEEE International Conference on Machine Learning and Applications (ICMLA '12), vol. 2, pp. 216-221, IEEE, December 2012.

[49] A. Alarifi, M. Alsaleh, A. Al-Salman, A. Alswayed, and A. Alkhaledi, "Google penguin: evasion in non-english languages and a new classifier," in Proceedings of the 12th International
Conference on Machine Learning and Applications (ICMLA '13), vol. 2, pp. 274-280, IEEE, December 2013.

[50] M. R. Stytz and S. B. Banks, "Dynamic software security testing," IEEE Security \& Privacy, no. 3, pp. 77-79, 2006.

[51] M. Alsaleh, A. Alqahtani, A. Alarifi, and A. Al-Salman, "Visualizing PHPIDS $\log$ files for better understanding of web server attacks," in Proceedings of the 10th Workshop on Visualization for Cyber Security (VizSec '13), pp. 1-8, October 2013.

[52] M. Alsaleh, A. Alarifi, A. Alqahtani, and A. Al-Salman, "Visualizing web server attacks: patterns in PHPIDS logs," Security and Communication Networks, vol. 8, no. 11, pp. 1991-2003, 2015.

[53] M. Curphey and R. Arawo, "Web application security assessment tools," IEEE Security \& Privacy, vol. 4, no. 4, pp. 32-41, 2006.

[54] Z. Alshaikh, A. Alarifi, and M. Alsaleh, "Christopher Alexander's fifteen properties: toward developing evaluation metrics for security visualizations," in Proceedings of the 11th IEEE International Conference on Intelligence and Security Informatics (IEEE ISI '13), pp. 295-300, IEEE, June 2013.

[55] N. Alomar, M. Alsaleh, and A. Alarifi, "Social authentication applications, attacks, defense strategies and future research directions: a systematic review," IEEE Communications Surveys \& Tutorials, vol. PP, no. 99, 2017.

[56] B. Arkin, S. Stender, and G. McGraw, "Software penetration testing," IEEE Security \& Privacy, vol. 3, no. 1, pp. 84-87, 2005.

[57] J. Bau, E. Bursztein, D. Gupta, and J. Mitchell, "State of the art: automated black-box web application vulnerability testing," in add Proceedings of the 31st IEEE Symposium on Security and Privacy (SP '10), pp. 332-345, IEEE, May 2010.

[58] N. Suteva, D. Zlatkovski, and A. Mileva, "Evaluation and testing of several free/open source web vulnerability scanners," in Proceedings of the 10th Conference for Informatics and Information Technology (CIIT '13), pp. 221-224, 2013.

[59] A. Austin, C. Holmgreen, and L. Williams, "A comparison of the efficiency and effectiveness of vulnerability discovery techniques," Information and Software Technology, vol. 55, no. 7, pp. 1279-1288, 2013.

[60] V. Felmetsger, L. Cavedon, C. Kruegel, and G. Vigna, “Toward automated detection of logic vulnerabilities in web applications," in Proceedings of the USENIX Security Symposium, pp. 143-160, 2010.

[61] Y.-W. Huang, C.-H. Tsai, T.-P. Lin, S.-K. Huang, D. T. Lee, and S.-Y. Kuo, "A testing framework for Web application security assessment," Computer Networks, vol. 48, no. 5, pp. 739-761, 2005.

[62] M. Finifter and D. Wagner, "Exploring the relationship between Web application development tools and security," in Proceedings of the USENIX Conference on Web Application Development, 2011.

[63] J. Fonseca and M. Vieira, "Mapping software faults with web security vulnerabilities," in Proceedings of the IEEE International Conference on Dependable Systems and Networks with FTCS and DCC, pp. 257-266, IEEE, June 2008.

[64] K. Soska and N. Christin, "Automatically detecting vulnerable websites before they turn malicious," in Proceedings of the USENIX Security, 2014.

[65] R. Sekar, "An efficient black-box technique for defeating web application attacks," in Proceedings of the 16th Annual Network and Distributed System Security Symposium (NDSS '09), 2009.

[66] A. Alarifi, M. Alsaleh, and N. Alomar, "A model for evaluating the security and usability of e-banking platforms," Computing, vol. 99, no. 5, pp. 519-535, 2017. 


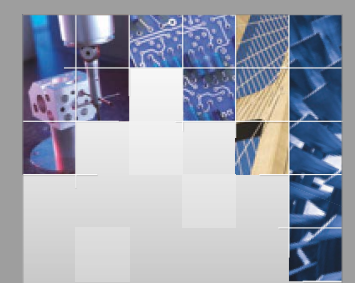

\section{Enfincering}
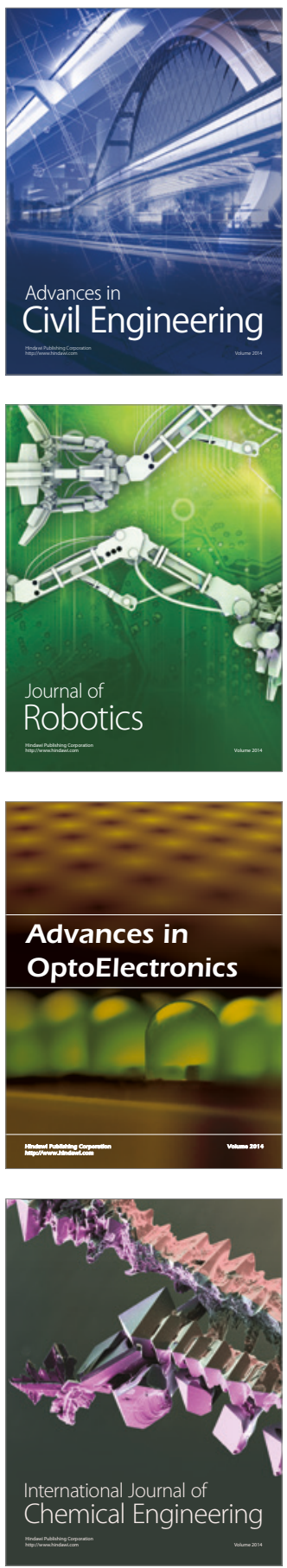

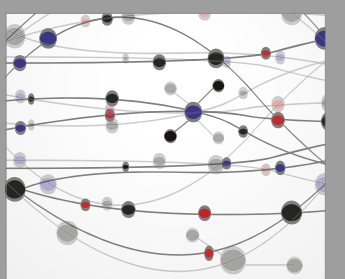

The Scientific World Journal

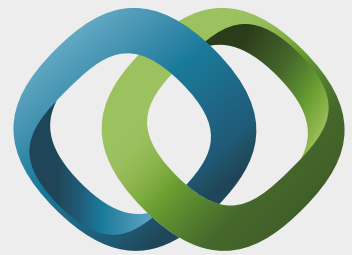

\section{Hindawi}

Submit your manuscripts at

https://www.hindawi.com
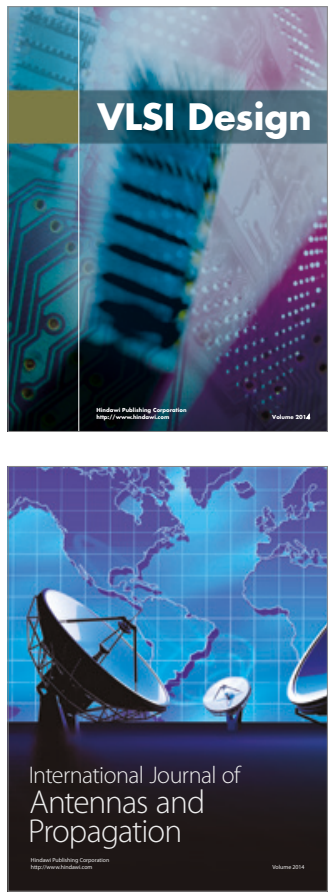

\section{Rotating}

Machinery
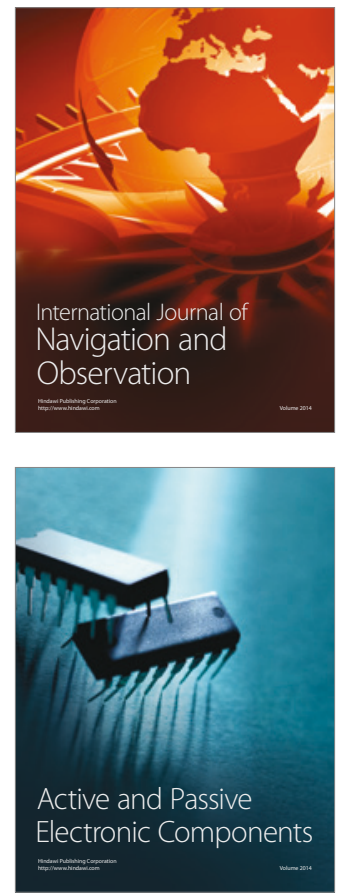
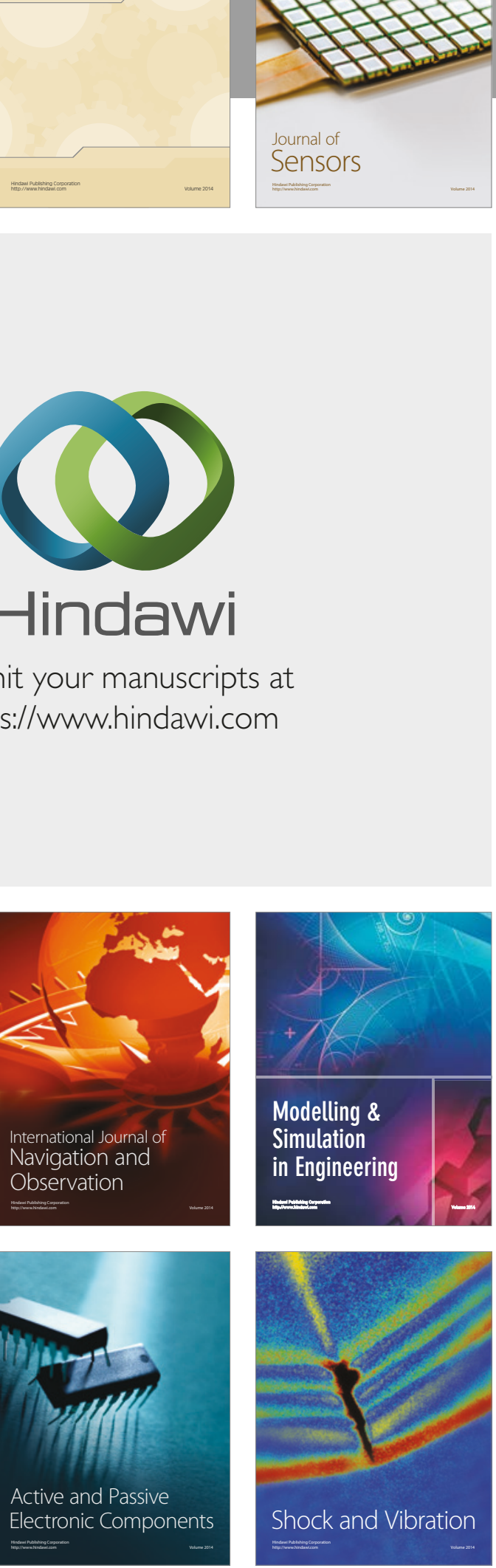
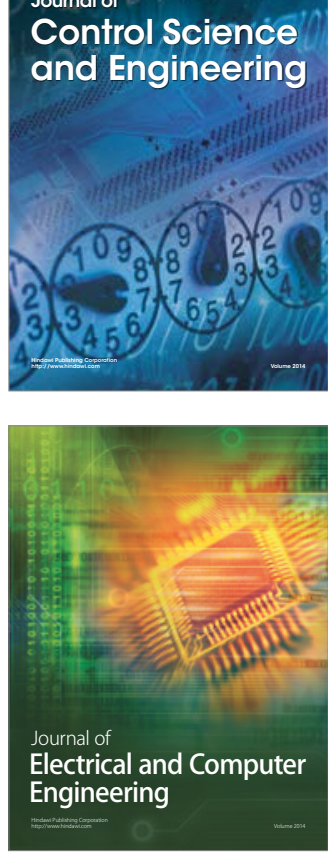

Distributed

Journal of

Control Science

and Engineering
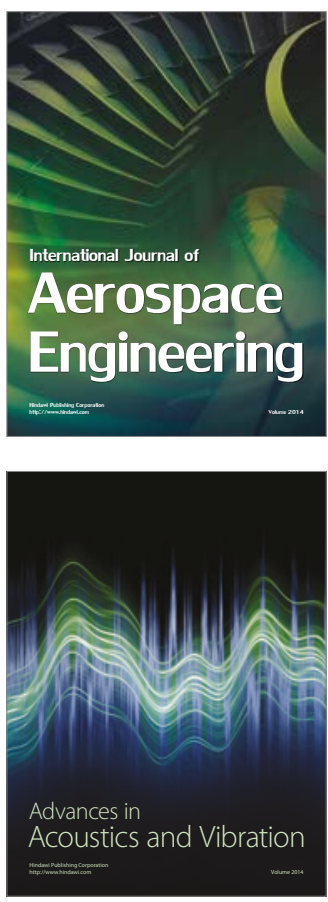

Sensor Networks 